\title{
Controle do Tempo de Protrombina em Sangue Capilar e Venoso em Pacientes com Anticoagulação Oral: Correlação e Concordância
}

\author{
Comparative Study of a Portable System for Prothrombin Monitoring Using Capillary Blood against Venous Blood \\ Measurements in Patients Using Oral Anticoagulants: Correlation and Concordance

\begin{abstract}
Tiago Luiz Luz Leiria, Lúcia Campos Pellanda, Eros Magalhães, Gustavo Glotz de Lima
Serviço de Eletrofisiologia/Ambulatório de Anticoagulação - Instituto de Cardiologia do Rio Grande do Sul - Fundação Universitária de Cardiologia, Porto Alegre, RS - Brasil
\end{abstract}

\section{Resumo}

Fundamento: $\mathrm{O}$ uso de anticoagulantes orais (ACO) é comum na prática cardiológica, tendo como principais indicações a fibrilação atrial e próteses valvares. Os pacientes em uso de ACO necessitam de controle freqüente do tempo de protrombina (TP). Novos sistemas portáteis de monitorização, para medida do tempo de protrombina, sem necessidade da coleta de sangue por venopunção, facilitam a rotina desses pacientes.

Objetivo: Comparar as medidas do TP realizadas pelo sistema Coaguchek $\mathbf{S}^{\circledR}$, em sangue capilar, com o método padrão em sangue venoso.

Métodos: Cento e vinte e sete pacientes em acompanhamento no ambulatório de anticoagulação do Instituto de Cardiologia foram submetidos ao método convencional e coleta de sangue capilar da polpa digital para medida com o sistema Coaguchek $\mathbf{S}^{\circledR}$.

Resultados: A idade foi de $58 \pm 14$ anos, $90 \%$ eram brancos. As indicações de ACO foram fibrilação atrial $49,6 \%$ e próteses valvares $37,0 \%$. O coeficiente de correlação $r_{s}$ foi $0,90(p<0,0001 ;$ IC:95\% 0,87-0,93) entre o sistema em estudo e o controle. A medida de concordância entre pacientes com INR <2, entre 2 e 3,5 e > 3,5 foi de Kappa 73,5\%. O sistema Coaguchek $S^{\circledR}$ superestimou o INR em 0,15 $\pm 0,85$ unidades. Valores acima de 3,5 unidades de INR mostram discrepância importante entre as duas técnicas.

Conclusões: $O$ sistema Coaguchek $\mathbf{S}^{\circledR}$ mostrou boa correlação e bom grau de concordância quando comparado com o controle, com resultados menores que 4. Porém, valores de INR acima de 3,5 ainda necessitam de confirmação com o método padrão. (Arq Bras Cardiol 2007; 89(1) : 1-5)

Palavras-chave: Anticoagulantes, protrombina, cumarínicos, tempo de protrombina.

\section{Summary}

Background: Oral anticoagulants (OAC) are widely used in cardiology and are mainly indicated in cases of atrial fibrillation and prosthetic heart valves. Regular prothrombin time (PT) control is required for patients using OAC. New portable monitoring systems for measuring prothrombin time, eliminate the need to collect blood by venous puncture and facilitate daily life for these patients.

Objective: To compare PT measurements using the Coaguchek $S^{\text {TM }}$ system with capillary blood and the standard method in venous blood.

Methods: One hundred and twenty-seven patients from the Cardiology Institute's anticoagulation clinic underwent conventional blood collection and capillary blood collection via a finger prick for measurements using the Coaguchek $S^{\text {TM }}$ system.

Results: The mean age was $58 \pm 14$ years and 90\% of the patients were white. OAC indications were atrial fibrillation (49.6\%) and prosthetic heart valves (37.0\%). The correlation coefficient, $r_{s}$, was $0.90\left(p<0.0001\right.$; Cl:95\% 0.87-0.93) between the Coaguchek $S^{\mathrm{TM}}$ system and the control method. The Kappa measure of agreement among the patients with INR $<2$, INR between 2 and 3.5 and INR $>3.5$ was $73.5 \%$. The Coaguchek $S^{\mathrm{TM}}$ system overestimated INR by $0.15 \pm 0.85$ units. A great deal of discrepancy was found between the two techniques for INR values higher than 3.5 units.

Conclusion: The Coaguchek $S^{\mathrm{TM}}$ system when compared to the control method revealed good correlation and a high degree of agreement for results lower than 4 units. However, confirmation is required for INR values above 3.5 using the standard method. (Arq Bras Cardiol 2007; 89(1) : 1-5)

Key words: Anticoagulants; prothrombin; coumarins; prothrombin time.

Full texts in English - http://www.arquivosonline.com.br

Correspondência: Tiago Luiz Luz Leiria •

Serviço de Eletrofisiologia/Ambulatório de Anticoagulação - Av. Princesa Isabel, 370 - Unidade de Pesquisa - $90620-001$ - Porto Alegre, RS - Brasil

E-mail: editoração-pc@cardiologia.org.br, tiagoleiria@yahoo.com

Artigo recebido em 27/07/06; revisado recebido em 27/08/06; aceito em 12/01/07. 


\section{Introdução}

O tratamento de pacientes que necessitam de terapia com anticoagulantes orais (antagonistas da vitamina K, varfarina ou femprocumona) tornou-se mais fácil com o desenvolvimento de sistemas de monitorização portáteis para medida do tempo de protrombina ${ }^{1}$. Esses sistemas não necessitam de coleta venosa de sangue, sendo o teste feito por meio de punção da polpa digital, com coleta de sangue capilar, para análise em fita reagente e dispositivo digital ${ }^{2}$.

Esse tipo de teste possui grande facilidade de execução, podendo ser realizado em qualquer lugar, tanto no domicílio do paciente quanto em clínicas de atendimento primário ou hospitais ${ }^{3,4}$. Esses aparatos utilizam tecnologia de microfluidos, com vários métodos de detecção, para gerar uma medida do tempo de protrombina sem a necessidade de coleta de sangue venoso periférico.

Hoje existem vários dispositivos de controle do tempo de protrombina ${ }^{5}$. O Serviço de Trombofilias da Clínica Mayo já utiliza, para o controle dos pacientes em uso de anticoagulante, o sistema Coaguchek $\mathrm{S}^{\circledR}$ (Roche Diagnostics, Indianápolis, Ind, USA) ${ }^{6}$. Nenhum desses aparelhos foi comparado com medidas laboratoriais, de forma prospectiva em situações reais de uso, em nosso país.

O controle do efeito anticoagulante dos antagonistas da vitamina Ké usualmente realizado por intermédio da medida do tempo de protrombina venoso. Exige venopunção, técnicos habilitados para tal procedimento, tempo de transporte da amostra para o laboratório e técnicos em bioquímica para a análise da amostra.

O objetivo deste estudo é comparar o sistema de monitorização de tempo de protrombina Coaguchek $\mathrm{S}^{\circledR} \mathrm{com}$ o método padrão utilizado em um hospital de referência em cardiologia, em pacientes acompanhados em ambulatório de anticoagulação especializado.

\section{Métodos}

Foi realizado um estudo transversal, no período de abril de 2006, envolvendo 127 pacientes consecutivos, em uso de anticoagulantes orais e acompanhados no ambulatório de anticoagulação do Instituto de Cardiologia do Rio Grande do Sul (IC/FUC), um hospital terciário de referência em cardiologia no Sul do país. Nesse ambulatório especializado são acompanhados aproximadamente 1.200 pacientes adultos e pediátricos por mês.

Os pacientes foram submetidos a coleta sangüínea realizada por venopunção para a realização do teste do tempo de protrombina no laboratório de análises clínicas de nossa Instituição, e, simultaneamente, a coleta de sangue capilar da polpa digital para medida no sistema Coaguchek $\mathrm{S}^{\circledR}$. As amostras foram recoletadas em caso de erros pré-analíticos (punção da polpa digital ineficaz, sangue aplicado de forma errônea na fita reagente).

O sistema de ponta Coaguchek $\mathrm{S}^{\circledR}$ é um dispositivo portátil, alimentado por baterias, que consiste de um fotômetro laser. Para realização do teste, uma gota de sangue capilar, de aproximadamente $10 \mu \mathrm{l}$, foi coletada da polpa do dedo indicador dos pacientes. O dispositivo com lanceta, SoftCLix
(Boehringer-Manheim, Germany), foi o instrumento utilizado para punção da polpa digital. A gota foi, então, depositada em fitas reagentes contendo tromboplastina e partículas de ferro que se misturaram com o sangue. No instante em que o sangue inicia o processo de coagulação, a atividade das partículas de óxido férrico diminui até o momento em que elas ficam completamente imóveis. As mudanças na mobilidade foram medidas por fotometria para determinação do tempo de protrombina.

O método convencional foi realizado por meio de venopunção com retirada de $10 \mathrm{ml}$ de sangue venoso em tubo de ensaio com citrato de sódio 3,2\%, o qual, após a coleta, foi imediatamente enviado para o laboratório central da Instituição. A dosagem do tempo de protrombina venoso foi executada no laboratório com tromboplastina humana, Thromborel $S^{\circledR}$ (Dade Behring, Newark, USA), em sistema automatizado CA-500 (Sysmex Corporation, Kobe, Japan), com valores de $I S I=1$. As preparações de tromboplastina foram utilizadas conforme recomendação da Organização Mundial de Saúde.

A análise estatística foi realizada no SPSS v. 12 SPSS inc e Medcalc v.8.2. Os dados contínuos de mensuração plasmática e capilar do tempo de protrombina em unidades de "international normalized ratio" (INR) foram analisados com uso do coeficiente de Spearman $\left(r_{s}\right)$. Esses dados, de mensuração venosa e capilar do tempo de protrombina na forma de INR, foram também transformados em ranks para análise da diferença entre os valores obtidos e o erro padrão.

A diferença entre os métodos estudados (bias) foi comparada com a média das duas medidas, demonstrada de forma gráfica conforme o método de Bland-Altman ${ }^{7}$, para evidenciar tendências e erros sistemáticos. Os limites de concordância entre as duas técnicas foram calculados como a diferença das médias $\pm 1,96$ desvio padrão. Para avaliar a concordância entre pacientes para variável sob a forma categórica (INR $<2$, entre 2 e 3,5 e > 3,5) foi utilizado o índice Kappa ${ }^{8}$.

O tamanho amostral estimado para detectar um $r_{\mathrm{s}}=0,9$, com erro alfa de $5 \%$ e beta de $20 \%$, seria de sete medidas com cada método; porém, para realização do método de Bland e Altman, conforme orientação dos autores, um tamanho amostral maior de 100 é indicado 9 . O protocolo de pesquisa foi aprovado no Comitê de Ética e Pesquisa de nosso hospital, todos os pacientes concordaram com termo de consentimento informado para participação no estudo.

\section{Resultados}

As características da população em estudo podem ser observadas na tabela 1. A principal indicação da necessidade do uso de anticoagulação oral na população em estudo foi de fibrilação atrial em 63 pacientes. As demais indicações podem ser vistas na tabela 2. Cinqüenta e cinco por cento dos pacientes $(n=70)$ apresentavam INR em níveis terapêuticos no dia da coleta sangüínea.

Nos 127 pacientes estudados, 6,3\% $(n=8)$ necessitaram repetir a coleta por punção da polpa digital, em razão de erros pré-analíticos (gota de sangue pequena e aplicação indevida do sangue na fita regente). 


\section{Artigo Original}

\begin{tabular}{|lc}
\hline \multicolumn{2}{|c}{ Tabela 1 - Características da população em estudo } \\
\hline Características & Pacientes (n=127) \\
\hline Sexo masculino & $55 \%$ \\
\hline Idade & $58 \pm 14$ \\
\hline Raça caucasianos & $90 \%$ \\
\hline Anticoagulante em uso & \\
\hline Femprocumona & $61 \%$ \\
\hline Varfarina & $39 \%$ \\
\hline Dose total semanal & $20 \mathrm{mg} \pm 0,5$ \\
\hline Femprocumona & $14 \pm 7$ \\
\hline Varfarina & $29 \pm 12$ \\
\hline Tempo de uso ACO meses & $36 *(1-240)$ \\
\hline ACO - anticoagulante oral, ${ }^{*}$ mediana. \\
\hline
\end{tabular}

\begin{tabular}{|c|c|}
\hline \multicolumn{2}{|c|}{ Tabela 2 - Indicações da anticoagulação oral } \\
\hline Indicação & Pacientes $(n=127)$ \\
\hline FA & $49,6 \%(63)$ \\
\hline Prótese metálica & $37 \%(47)$ \\
\hline TEP & $2,4 \%(3)$ \\
\hline TVP & $0,8 \%(1)$ \\
\hline IAM & $3,1 \%(4)$ \\
\hline $\mathrm{IC}$ & $2,4 \%(3)$ \\
\hline Outros & $4,7 \%(6)$ \\
\hline $\begin{array}{l}\text { FA- fibrilação atrial; } T \\
\text { venosa profunda; IAN } \\
\text { cardíaca. }\end{array}$ & $\begin{array}{l}\text { no pulmonar; TVP - tromb } \\
\text { miocárdio; IC - insuficiêr }\end{array}$ \\
\hline
\end{tabular}

As distribuições dos valores de INR medidos com o sistema em estudo e o controle mostraram distribuição assimétrica. A correlação entre a medida do INR com o sistema Coaguchek $\mathrm{S}^{\circledR}$ e a medida padrão de nosso laboratório pode ser vista na figura 1. O coeficiente de Spearman $\left(r_{\mathrm{s}}\right)$ foi de $0,90(\mathrm{P}<0,0001$; IC:95\% 0,87-0,93) entre o sistema em estudo e o controle.

O INR médio com o sistema Coaguchek $\mathrm{S}^{\circledR}$ foi de $2,75 \pm 1,40$ unidades INR. O valor médio do INR com o método convencional foi de 2,59 $\pm 1,41$ unidades INR. O sistema Coaguchek $\mathrm{S}^{\circledR}$ superestimou os valores de INR em 0,15 unidades em todos os níveis de medida (IC:95\% $=0,007$ - 0,309). A figura 2 faz a representação gráfica dessa diferença (bias), quando comparada com a média das duas medidas, sistema Coaguchek $\mathrm{S}^{\circledR}$ e laboratório. Nessa figura são apresentadas a margem de erro dos valores medidos com o sistema de análise, do tempo de protrombina, capilar e o intervalo de confiança.

A tabela 3 mostra a diferença média (bias) e o desvio padrão entre diferentes estratos de INR; menor que 2, entre 2 e 3 , entre 3,01 e 3,5 e maior que 3,51 unidades de INR.

Os valores de INR variaram de 1,01 até 10, baseados

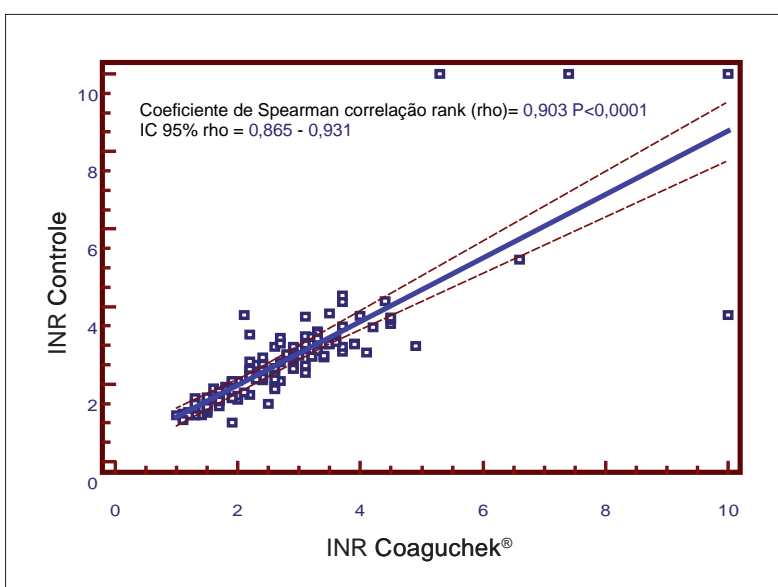

Fig. 1 - Gráfico da correlação entre a medida dos INR entre o sistema Coaguchek $S^{\circledR}$ e o controle.

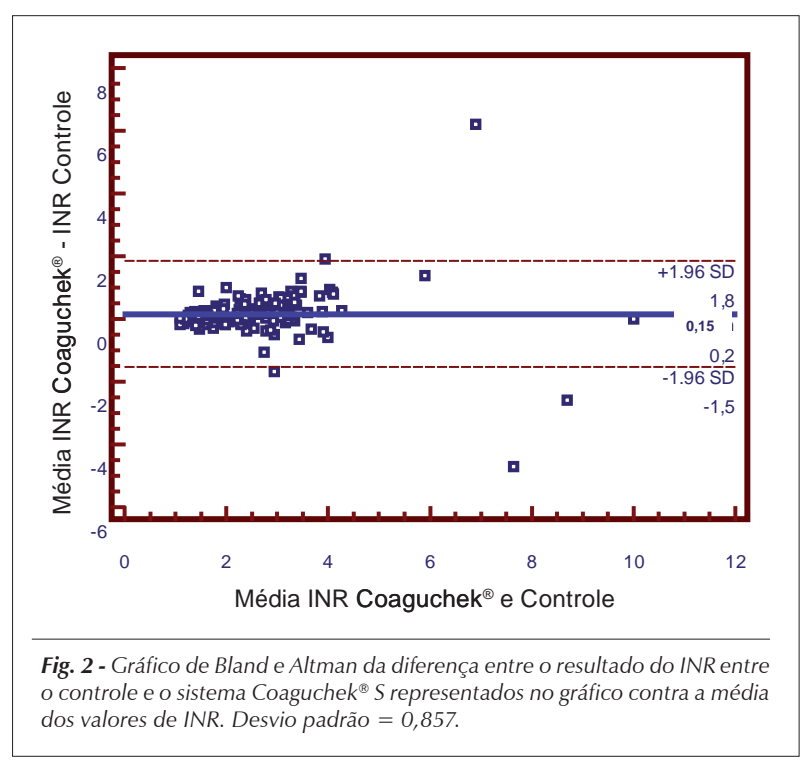

Tabela 3 - Diferença média entre o INR medido com sistema Coguchek $S^{\circledR}$ e o controle e margem de erro

\begin{tabular}{|lccc|}
\hline Categoria INR & $\mathbf{n}$ & Diferença & Erro padrão \\
\hline$<2$ & 42 & 0,12 & 0,29 \\
\hline $2,01-3,0$ & 50 & 0,25 & 0,42 \\
\hline $3,01-3,5$ & 20 & 0,15 & 0,56 \\
\hline 3,51 & 15 & 0,01 & 2,32 \\
\hline $\begin{array}{l}\text { Médio todas } \\
\text { categorias }\end{array}$ & 127 & 0,15 & 0,85 \\
\hline
\end{tabular}

no método de controle realizado em nossa Instituição. Fundamentado nesses dados, 55,1\% (70/127) dos pacientes estariam com INR em níveis terapêuticos; 33,1\% (42/127) subanticoagulados, e 11,8\% (15/127) com níveis de anticoagulação acima de 3,5 unidades de INR. Com o sistema 
Coaguchek $\mathrm{S}^{\circledR}, 52,1 \%$ (67/127) dos pacientes estariam com INR em níveis terapêuticos; 29,9\% (38/127) subanticoagulados, e $17,8 \%$ (22/127) com níveis de anticoagulação acima de 3,5 unidades de INR. O índice de concordância Kappa foi de $73,5 \%$ (tab. 4).

Em relação às decisões clínicas baseadas no novo sistema, quando comparadas com o método controle, essas levariam a um ajuste indevido de dose em 23 casos (18\%). Em sete pacientes, seria necessário aumentar as doses de anticoagulante, mas isso não ocorreria se fosse considerado o intervalo de 2 a 3,5 unidades INR obtido no Coagucheck $\mathrm{S}^{\circledR}$. Três casos teriam a dose da medicação aumentada, já estando em níveis terapêuticos, 10 casos teriam a dose reduzida sem necessidade e três pacientes teriam sua dose mantida, porém estando com valores de INR acima de 3,5 unidades (tab. 4).

\section{Discussão}

Os anticoagulantes orais possuem uma grande variação na relação entre dose e resposta terapêutica. Isso faz com que seja necessária a monitorização freqüente do tempo de protrombina dos pacientes em uso desse tipo de medicação.

A forma recomendada do controle do tempo de protrombina é a de utilização do INR. Relatar o resultado dessa forma facilita a comparação entre medidas realizadas com diferentes sistemas e laboratórios ${ }^{10}$.

O desenvolvimento de sistemas portáteis de controle do tempo de protrombina oferece vantagens importantes no manejo dessa população de pacientes ${ }^{5,11}$. Escolher um sistema que se mostre confiável é de suma importância para que a medida seja a mais precisa possível.

Para o estudo, consideramos que a maneira mais precisa de realizar o controle do tempo de protrombina é o método utilizado em nosso laboratório central, o qual utiliza tromboplastina humana com alta sensibilidade $(I S I=1)$, conforme recomendado pelo Colégio Americano de Patologia ${ }^{12}$.

Diversos estudos já evidenciaram existir boa correlação entre o sistema Coaguchek $S^{\circledR}$ e medidas laboratoriais ${ }^{3,13,14}$. Centros médicos, como a Clínica Mayo, já utilizam a tecnologia desses dispositivos no manejo de seus pacientes ${ }^{6}$. Essa forma de controle do tempo de protrombina ainda não havia sido testada em nosso meio.

A correlação entre valores de INR, medidos entre o sistema em estudo e o controle, mostrou-se quase perfeita ${ }^{15}$, com coeficiente $r=0,9$, semelhante ao encontrado em outros estudos envolvendo o Coaguchek $S^{\circledR}$ e outros dispositivos de controle do tempo de protrombina ${ }^{6,16,17}$. Porém, conforme Bland e Altman ${ }^{7}$, a melhor maneira de avaliar medidas entre dois métodos não é a utilização de coeficientes de correlação, pois esses apenas informam a variação dos dois resultados e não as diferenças absolutas. Assim, dois exames podem apresentar uma correlação perfeita, apesar de uma diferença sistemática e significativa entre os métodos. A forma proposta de melhor descrever o resultado seria a diferença das médias incluindo a margem de erro.

Em nosso estudo, o sistema Coaguchek $\mathrm{S}^{\circledR}$ superestimou as medidas do INR em 0,15 unidades, com desvio padrão de 0,85 unidades de INR. Além disso, o desvio padrão dos valores aumenta quanto maior for o INR. As medidas de INR são muito confiáveis em valores de INR entre 1 e 2, confiáveis entre 3,01 e 3,5, e relativamente confiáveis entre 2,01 e 3 .

Estudos prévios já haviam feito essa consideração ${ }^{6,16}$. O sistema Coaguchek $\mathrm{S}^{\circledR}$ perde qualidade quando comparado com medidas laboratoriais usando tromboplastinas sensíveis, nos valores de INR maiores que 4. Estudo feito na Cleveland Clinic $^{18}$, utilizando método de controle laboratorial semelhante ao utilizado em nosso hospital (sistema analisador de coagulação Sysmex e tromboplastina humana Dade Behring com $I S I=1$ ), também mostrou alteração em valores de INR quando esses eram maiores do que 4. Essa pesquisa avaliou dois outros dispositivos, o AvoSure PT ProSystem (AvoSure device; Avocet Medical Inc., San Jose, CA) e o ProTime Microcoagulation System (ProTime device; International Technidyne Corporation Limited, Edison, NJ). Os bias para os sistemas ProTime e AvoSure foram de 0,5 $\pm 0,4$ e de 0,4 $\pm 0,5$ unidades de INR, respectivamente, sendo esses valores superiores ao encontrado no sistema Coaguchek $\mathrm{S}^{\circledR}$ em nosso estudo $(0,2 \pm 0,8)$.

Em relação à concordância dos resultados nos intervalos de 1-2, 2-3,5 e > 3,5 o índice Kappa foi de 73,5\%, que significa concordância substancial de acordo com a literatura ${ }^{19}$. Porém, mudanças nas decisões clínicas ocorreriam em 18\% dos casos em nosso estudo se essas fossem realizadas com base apenas no sistema portátil. Esse valor cairia para $10 \%$ se os resultados acima de 3,5 INR, medidos com dispositivo Coaguchek $\mathrm{S}^{\circledR}$, fossem repetidos no laboratório central. Esse valor estaria de acordo com trabalho publicado por Chapman e cols. ${ }^{20}$, no qual um índice de erro de $8 \%$ ocorreu nas decisões clínicas baseadas no sistema Coaguchek $\mathrm{S}^{\circledR}$.

Estudos clínicos, comparando a segurança desse dispositivo, em termos da ocorrência de desfechos relevantes (incidência

\begin{tabular}{|c|c|c|c|c|c|}
\hline & INR Coaguchek & INR $<2$ & INR $=2-3,5$ & INR $>3,5$ & Total (\%) \\
\hline \multicolumn{6}{|l|}{ Controle } \\
\hline INR $<2$ & & 35 & 7 & 0 & $42(33,1)$ \\
\hline INR $2-3,5$ & & 3 & 57 & 10 & $70(55,1)$ \\
\hline INR > 3,5 & & 0 & 3 & 12 & $15(11,8)$ \\
\hline Total (\%) & & $38(29,9)$ & $67(52,8)$ & $22(17,3)$ & 127 \\
\hline
\end{tabular}


de sangramentos, incidência de embolia), ainda não foram realizados. Contudo, o uso desse tipo de dispositivo em clínicas de anticoagulação e para autocontrole feito pelo próprio paciente é comum na Inglaterra ${ }^{21}$.

O sistema Coaguchek $\mathrm{S}^{\circledR}$ possui boa concordância com os valores laboratoriais para as duas principais indicações de anticoagulação oral, prótese valvar e fibrilação atrial. Segundo Cannegieter e cols. ${ }^{22}$, analisando mais de 1.600 pacientes, em clínica de anticoagulação na Holanda (portadores de prótese valvar), a incidência de fenômenos hemorrágicos e eventos embólicos foi menor no intervalo entre 2,5 até 4,9 unidades de INR. A utilização do dispositivo Coaguchek $S^{\circledR}$ no controle do tempo de protrombina nos pacientes com fibrilação atrial parece-nos segura. Isso pelo fato de que portadores dessa arritmia devem manter o valor alvo de INR entre 2 e 3 . Os que apresentam níveis de INR abaixo de 2 possuem uma razão de chance de 1,9 para ocorrência de eventos embólicos. A incidência de eventos hemorrágicos é de três eventos por 100 pacientes/ano naqueles em que o INR fica acima de $4^{23}$.

As potenciais limitações de nosso estudo encontram-se no fato de termos incluídos pacientes com valores de INR até 10 (grande parte dos estudos exclui pacientes com INR > 7), pois já era sabido que resultados elevados de INR tendem a aumentar a discrepância entre as medidas; as duas medidas em cada método para analisar reprodutibilidade não foram realizadas.

Com base nos dados de nosso estudo, tem-se que o sistema Coaguchek $\mathrm{S}^{\circledR}$ pode ser utilizado na monitorização do tempo de protrombina em pacientes com uso de anticoagulantes orais em ambulatório de anticoagulação, desde que valores acima de 3,5 unidades de INR sejam confirmados por meio de medidas realizadas no laboratório central.

A realização de ensaio clínico randomizado alocando pacientes para o controle do tempo de protrombina com o sistema Coaguchek $\mathrm{S}^{\circledR}$ e outro grupo com controle por meio do laboratório, tendo como desfechos eventos clínicos relevantes (exemplo: sangramento, AVC) e o tempo no intervalo terapêutico, parece-nos a melhor maneira de avaliar a segurança e a efetividade desse dispositivo para o uso disseminado.

\section{Potencial Conflito de Interesses}

Declaro não haver conflitos de interesses pertinentes.

\section{Referências}

1. Douketis JD. Patient self-monitoring of oral anticoagulant therapy: potential benefits and implications for clinical practice. Am J Cardiovasc Drugs. 2001; 1: 245-51.

2. Machin SJ, Mackie IJ, Chitolie A, Lawrie AS. Near patient testing (NPT) in haemostasis - a synoptic review. Clin Lab Haematol. 1996; 18: 69-74.

3. Shiach CR, Campbell B, Poller L, Keown M, Chauhan N. Reliability of point-ofcare prothrombin time testing in a community clinic: a randomized crossover comparison with hospital laboratory testing. Br J Haematol. 2002; 119: 370-5.

4. Heneghan C, Alonso-Coello P, Garcia-Alamino JM, Perera R, Meats E, Glasziou P. Self-monitoring of oral anticoagulation: a systematic review and meta-analysis. Lancet. 2006; 367: 404-11.

5. Murray ET, Fitzmaurice DA, McCahonD. Point of care testing for INR monitoring: where are we now? Br J Haematol. 2004; 127: 373-8.

6. McBane RD 2nd, Felty CL, Hartgers ML, Chaudhry R, Beyer LK, Santrach PJ. Importance of device evaluation for point-of-care prothrombin time international normalized ratio testing programs. Mayo Clin Proc. 2005; 80 (2): 181-6.

7. Bland JM, Altman DG. Statistical methods for assessing agreement between two methods of clinical measurement. Lancet. 1986; i: 307-10.

8. Landis JR, Koch GG. The measurement of observer agreement for categorical data. Biometrics. 1977; 33: 159-74.

9. Bland JM, Altman DG. Comparing methods of measurement - why plotting difference against standard method is misleading. Lancet. 1995; 346: 1085-7.

10. Hirsh J, Poller L. The international normalized ratio. A guide to understanding and correcting its problems. Arch Intern Med. 1994; 154 (3): 282-8.

11. Murray ET, Greaves M. INRs and point of care testing. BMJ. 2003; 327: 5-6.

12. Fairweather RB, Ansell J, van den Besselaar AM, Brandt, Bussey HI, Poller $\mathrm{L}$, et al. College of American Pathologists Conference XXXI on laboratory monitoring of anticoagulant therapy: laboratory monitoring of oral anticoagulant therapy. Arch Pathol Lab Med. 1998; 122 (9): 768-81.
13. Oberhardt BJ, Dermott SC, Taylor M, Alkadi ZY, Abruzzini AF, Gresalfi NJ. Dry reagent technology for rapid, convenient measurements of blood coagulation and fibrinolysis. Clin Chem. 1991; 37: 520-6.

14. Rose VL, Dermott SC, Murray BF, Mclver MM, High KA, Oberhardt $B J$. Decentralized testing for prothrombin time and activated partial thromboplastin time using a dry chemistry portable analyzer. Arch Pathol Lab Med. 1993; 117: 611-7.

15. McGraw KO, Wong SP. A common language effect-size statistic. Psychol Bull. 1992; 111: 361-5.

16. Havrda DE, Hawk TL, Marvin CM. Accuracy and precision of the Coaguchek $S^{\circledR}$ versus laboratory INRs in a clinic. Ann Pharmacother. 2002; 36 (5): 769-75.

17. Vacas M, Lafuente PJ, Unanue I, Iriarte JA. Comparative study of two portable systems for oral anticoagulant monitoring. Hematol J. 2004; 5 (1): 35-8.

18. Shermock KM, Bragg L, Connor JT, Fink J, Mazzoli G, Kottke-Marchant K. Differences in warfarin dosing decisions based on international normalized ratio measurements with two point-of-care testing devices and a reference laboratory measurement. Pharmacotherapy. 2002; 22 (11): 1397-404.

19. Landis JR, Koch GG. The measurement of observer agreement for categorical data. Biometrics. 1977; 33: 159-74.

20. Chapman DC, Stephens MA, Hamann GL, Bailey LE, Dorko CS.Accuracy, clinical correlation, and patient acceptance of two handheld prothrombin time monitoring devices in the ambulatory setting. Ann Pharmacother. 1999; 33 (7-8): 775-80.

21. Heneghan C, Alonso-Coello P, Garcia-Alamino J, Perera R, Meats E, Glasziou P Self-monitoring of oral anticoagulation: a systematic review and metaanalysis. Lancet. 2006; 367 (9508): 404-11.

22. Cannegieter SC, Rosendaal FR, Wintzen AR, van der Meer FJM, Vandenbroucke JP, Briet E. Optimal oral anticoagulant therapy in patients with mechanical heart valves. N Engl J Med. 1995; 333: 11-7.

23. Hylek EM, Go AS, Chang Y, Jensvold NG, Henault LE, Selby JV, et al. Effect of intensity of oral anticoagulation on stroke severity and mortality in atrial fibrillation. N Engl J Med. 2003; 349: 1019-26. 